\title{
Luminescence dynamics of bound exciton of hydrogen doped ZnO nanowires
}

\author{
Jinkyoung Yoo ${ }^{a,{ }^{*}}$, Gyu-Chul Yi ${ }^{b,{ }^{*}}$, Bonghwan Chon ${ }^{\mathrm{c}, \S}$, Taiha Joo ${ }^{c}$, Zhehui Wang $^{\mathrm{d}}$ \\ ${ }^{a}$ Center for Integrated Nanotechnologies, Los Alamos National Laboratory, Los Alamos, NM 87545, USA \\ ${ }^{b}$ Department of Physics and Astronomy, and Institute of Applied Physics, Seoul National University, Seoul 151747, Republic of \\ Korea \\ ${ }^{c}$ Department of Chemistry, POSTECH, Pohang 790784, Republic of Korea \\ ${ }^{\S}$ Current address: Center for Molecular Spectroscopy and Dynamics, Institute for Basic Science, Seoul 136-713, Republic of \\ Korea, Department of Chemistry, Korea University, Seoul 136-713, Republic of Korea \\ ${ }^{d} P-25$, Los Alamos National Laboratory, Los Alamos, NM 87545, USA
}

\section{ABSTRACT}

All-optical camera, converting X-rays into visible photons, is a promising strategy for high-performance X-ray imaging detector requiring high detection efficiency and ultrafast detector response time. Zinc oxide is a suitable material for all-optical camera due to its fast radiative recombination lifetime in sub-nanosecond regime and its radiation hardness. ZnO nanostructures have been considered as proper building blocks for ultrafast detectors with spatial resolution in sub-micrometer scale. To achieve remarkable enhancement of luminescence efficiency $n$-type doping in $\mathrm{ZnO}$ has been employed. However, luminescence dynamics of doped $\mathrm{ZnO}$ nanostructures have not been thoroughly investigated whereas undoped $\mathrm{ZnO}$ nanostructures have been employed to study their luminescence dynamics. Here we report a study of luminescence dynamics of hydrogen doped ZnO nanowires obtained by hydrogen plasma treatment. Hydrogen doping in $\mathrm{ZnO}$ nanowires gives rise to significant increase in the near-bandedge emission of $\mathrm{ZnO}$ and decrease in averaged photoluminescence lifetime from 300 to 140 ps at $10 \mathrm{~K}$. The effects of hydrogen doping on the luminescent characteristics of $\mathrm{ZnO}$ nanowires were changed by hydrogen doping process variables.

\section{Introduction}

X-ray imaging has revolutionized our understanding of matter in many ways. Hard X-rays with energies above 10 keV, like energetic electrons and protons, ${ }^{1,2}$ are highly penetrating and thus essential tools for imaging of macroscopic objects with 
microscopic (sub- $\mu \mathrm{m})$ resolution. X-ray imaging, unlike its charged-particle counterparts, is not limited by long-range Coulomb interactions. In other words, spatial resolutions of X-ray imaging does not degrade as the samples get thicker. X-ray free electron lasers (XFELs), also known as the 4th generation light source, are unique in ultrafast imaging of materials when sub- $\mu \mathrm{m}$ spatial and sub-ns temporal resolution are needed simultaneously. Significant advances in X-ray detectors, and imaging detectors in particular, are needed to fully realize the potentials of XFELs for ultrafast imaging of materials. ${ }^{3}$

Some of the known X-ray imaging detector challenges include high X-ray detection efficiency, picosecond detector response time, GHz framerate, and large data rate. Thus, it is worthwhile to explore new detector concepts, for example, an all-optical camera (AOC) for ultrafast hard X-ray imaging. An AOC converts X-rays into visible photons and employs photons rather than electrons throughout the chain of signal propagation and transmission. Replacing electrons by photons in image formation and transmission can potentially increase frame rate by several orders of magnitude, permits $\mathrm{GHz}$ frame rates as required by the ultrafast processes in material response.

A critical step toward the development of an AOC is demonstrating an optical frontend, which can be either conventional scintillators or newer light-emitting semiconductors. Zinc oxide $(\mathrm{ZnO})$ is known to be a fast scintillator, a sub-nanosecond decay constant was reported by Lehmann in 1966, and Luckey demonstrated a ZnO scintillator in $1968 .^{4-9}$ A key challenge with fast scintillators is maximizing light output without compromising spatial resolution. This can be achieved if the light can be channeled or propagated into a waveguide. ${ }^{10,11}$ From the perspective nanowires (NWs) can be ideal building blocks for high-spatial resolution fast scintillators. ${ }^{7}$ Each ZnO NW acts as a scintillator column with sub-micrometer lateral dimensions, and the emitted light is guided along the ZnO NWs. Additionally ZnO NWs can be easily prepared by various methods such as chemical vapor deposition and hydrothermal synthesis. Nevertheless, ZnO NWs are not ideal for scintillator due to selfabsorbency problem, absorption of the luminescence through band-to-band transition inside $\mathrm{ZnO} \mathrm{NW}$ before light extraction. $^{5,9}$

With bulk $\mathrm{ZnO}$ crystals, the self-absorption can be addressed by red-shifting the dominant emission wavelength via $n$-type doping using gallium (Ga) incorporation. ${ }^{5,6,12} \mathrm{~N}$-type doping of $\mathrm{ZnO}$ induces remarkable increase in the population of bound excitons, the emission wavelength of which is longer than that of free excitons. Furthermore, the giant oscillator strength of bound excitons in $\mathrm{ZnO}$ results in a significant enhancement of the luminous intensity as well as a reduction in the luminescence decay constants. However the properties of Ga doping in $\mathrm{ZnO}$ NWs are not identical to those in bulk and in thin films. Dopants in nanostructures tend to segregate either at the surface or at specific crystal planes causing changes in the growth direction of the NWs, and leading to the formation of structural defects. ${ }^{12}$ Ga modulation doping with a spatially 
confined dopant distribution has been employed to overcome such structural deformations in NWs, ${ }^{13,14}$ but the process is not suitable for changing the wavelength of the dominant luminescent emission because the dopants are distributed in only a small part of the nanostructure.

Hydrogen is an alternative $n$-type dopant, and induces minimal structural deformation due to its small ionic radius. Hydrogen atoms act as donors only in $\mathrm{ZnO}$ though hydrogen atoms are usually amphoteric dopants compensating dominant charge carriers. ${ }^{15}$ Because hydrogen is a ubiquitous element and reliable in $\mathrm{ZnO}$, hydrogen incorporation in $\mathrm{ZnO}$ is facile, ${ }^{16-24}$ and has been studied extensively. Moreover, hydrogen can be diffused into bulk $\mathrm{ZnO}$ rapidly even at $100^{\circ} \mathrm{C} .{ }^{16}$ However, the luminescence dynamics of hydrogen doped ZnO NWs have not been thoroughly studied. Here we report a comprehensive study of the luminescence dynamics of hydrogen doped $\mathrm{ZnO} \mathrm{NWs}$ to elucidate luminescence mechanisms of $n$-type $\mathrm{ZnO}$ NWs for tuning luminescent characteristics.

\section{Experimental details}

Both as-grown and hydrogen-plasma treated ZnO NWs were prepared on $n$-type Si (100) substrates via the metal-organic chemical vaport deposition. Diethylzinc (DEZn) and oxygen were the reactants and their flow rates were in the range of 3-10 sccm and 50-100 sccm, respectively. Typical growth temperature was $500^{\circ} \mathrm{C}$. No metal catalyst was coated on the substrates during nanoneedle growth. The diameters of $\mathrm{ZnO}$ NWs were ranging from 50 to $70 \mathrm{~nm}$. Details of ZnO NW growth are reported elsewhere. ${ }^{25}$ Hydrogen doping in $\mathrm{ZnO}$ NWs was performed by radio frequency (rf) plasma treatments for 5 min with different plasma powers. The hydrogen flow rate was fixed at $200 \mathrm{sccm}$ resulting in $65 \mathrm{mTorr}$, and the rf plasma frequency was $13.56 \mathrm{MHz}$, and the rf power was varied in the range of 20 and $40 \mathrm{~W}$. The temperature of plasma chamber increased up to $50^{\circ} \mathrm{C}$ for the plasma power of $30 \mathrm{~W}$. The luminescent characteristics of hydrogen plasma treated $\mathrm{ZnO} \mathrm{NWs}$ were investigated by time-integrated photoluminescence (TIPL) and time-resolved photoluminescence (TRPL) measurements at the temperatures ranging 10 to $200 \mathrm{~K}$. The sample was glued on the cold finger with cryogenic grease, and the sample temperature was controlled by closed cycle Helium refrigerator and a cartridge heater installed below the cold finger. The excitation sources of TIPL and TRPL were a continuous wave He-Cd laser ( $\lambda=325 \mathrm{~nm}$, Kimmon Koha Co.) and a frequencytripled Ti:Sapphire laser ( $\lambda=266 \mathrm{~nm}$, Tsunami, Spectra-Physics) with the pulse width of $100 \mathrm{fs}$ and the repetition rate of 82 MHz, respectively. The TIPL of the hydrogen plasma treated ZnO NWs was delivered into a monochromator (SPEX 750i, Jobin Yvon) with the spectral resolution of $0.15 \mathrm{~nm}$. The TIPL was measured by a photomultiplier tube (PMT) (R636-10, Hamamatsu). The TRPL was measured by a time-correlated single photon counting (TCSPC) system equipped with a 
monochromator (SP300, Acton) and a microchannel plate PMT (R3809-51, Hamamatsu). The time resolution and the spectral resolution of the TCSPC system were $\sim 8$ ps and $0.2 \mathrm{~nm}$, respectively.

\section{Results and Discussion}

\subsection{Effect of hydrogen plasma treatment on morphology and time-integrated PL spectra of ZnO NWs}

The morphology of the ZnO NWs following hydrogen plasma treatment was observed using scanning electron microscopy (SEM). Figures 1(a) and 1(b) show the ZnO NWs before and after hydrogen plasma treatment at $30 \mathrm{~W}$, respectively. The sharp tips of ZnO NWs shown in Fig. 1(a) indicate formations that minimize the area of the ZnO (0001) plane. As shown in Fig. 1(b), hydrogen plasma treatment did not induce morphological changes (note that hydrogen plasma power with a power greater than $40 \mathrm{~W}$ resulted in etching of the ZnO NWs). Figure 1(c) shows the PL spectra of the as prepared and plasmatreated ZnO NWs at room temperature (RT). The RT PL spectrum of as-prepared ZnO NWs exhibited strong near-band-edge (NBE) emission at $3.22 \mathrm{eV}$. Green emission originating from oxygen vacancies was not observable within the detection limit. Hydrogen plasma treatment at $30 \mathrm{~W}$ enhanced both the integrated and the peak RT PL intensity by 30\%, with no noticeable changes in the spectral shape. This enhancement of PL intensity of the hydrogen plasma-treated ZnO NWs is consistent with the previous findings, and has been explained either by hydrogen doping or by passivation of defect states. ${ }^{21-24,26-29}$ Since the RT PL spectrum of the as-prepared ZnO NWs did not exhibit visible emission, hydrogen doping can be considered as the main mechanism of the PL enhancement of the plasma-treated $\mathrm{ZnO}$ NWs.

Low temperature PL measurements can provide evidence of hydrogen doping. Figure 1(d) shows the $10 \mathrm{~K}$ TIPL spectra of the as-prepared $\mathrm{ZnO}$ at $10 \mathrm{~K}$, as well as those of the hydrogen plasma-treated ZnO NWs with different plasma powers. The $10 \mathrm{~K}$ PL spectrum of the as-prepared ZnO (dotted curve) exhibited free exciton emission at $3.376 \mathrm{eV}\left(\mathrm{X}_{\mathrm{A}}\right)$ indicating the high purity of the ZnO NWs. Such highly pure ZnO NWs are optimal platforms to study the effects of doping on the PL characteristics. The power used during the hydrogen plasma treatment significantly affects the dominant emission energy and spectral shape. With a plasma power of $20 \mathrm{~W}$, the integrated PL intensity over all the NBE emission wavelengths ranging from 365 to $380 \mathrm{~nm}$ increased with no notable changes in the spectral shape. A further increase in the power to $30 \mathrm{~W}$ resulted in a three-fold increase in integrated PL intensity, and the dominant emission changed to $3.363 \mathrm{eV}\left(\mathrm{I}_{4}\right)$. The $10 \mathrm{~K}$ PL spectrum of the hydrogen plasma-treated ZnO NWs at $30 \mathrm{~W}$ exhibited three emission peaks at $3.363\left(\mathrm{I}_{4}\right)$ and $3.353\left(\mathrm{I}_{10}\right)$ eV. The chemical identity of the $\mathrm{I}_{7}$ is unclear, but $\mathrm{I}_{4}$ can be assigned to hydrogen donor bound excitons, providing evidence of hydrogen doping in the ZnO NWs treated with $30 \mathrm{~W}$ plasma. The $10 \mathrm{~K}$ PL intensity of the hydrogen doped ZnO NWs treated at $40 \mathrm{~W}$ drastically decreased. Hydrogen reacts with $\mathrm{ZnO}$ under energetic conditions such as plasma treatment, so we may 
expect a sufficiently high-energy hydrogen plasma to lead to decomposition of $\mathrm{ZnO}$, resulting in surface damage. We have previously shown that ZnO NWs are etched when the hydrogen plasma power exceeds $40 \mathrm{~W} .{ }^{18}$ Damages to the surface due to the hydrogen plasma result in nonradiative recombination centers at the surfaces, and subsequently the PL intensity decreases significantly.

Hydrogen doping affects the physical properties of $\mathrm{ZnO}$ in two ways: surface passivation leading to an increase in the electron concentration in surface-depleted regions and changes in the dominant exciton recombination channels. ${ }^{21-24,26-29}$ Surface passivation effect of hydrogen doping is manifested by dramatic quenching of the visible PL emission from hydrogen doped ZnO thin films and NWs. Figure 1(c) shows negligible visible emission, which suggests that surface passivation with hydrogen can be studied by investigating thermal quenching of the PL. Figure 2(a) shows the temperature-dependent PL spectra of hydrogen doped ZnO NWs in the range of 10 to $300 \mathrm{~K}$. The $\mathrm{I}_{4}$ emission disappeared at around $120 \mathrm{~K}$, and the localization energy of the $\mathrm{I}_{4}$ emission of $13 \mathrm{meV}$ is consistent with that of hydrogen donor bound excitons. ${ }^{30}$ Figure 2(b) shows the relative integrated PL intensity over the entire measured wavelengths at various temperatures compared with that at $10 \mathrm{~K}$. The PL thermal quenching of hydrogen doped ZnO NWs at $30 \mathrm{~W}$ was more significant than that of the undoped (asprepared) ZnO NWs. For the undoped ZnO NWs, the surface band is bent upward in energy, due to trapping of free electrons at the surface states. Thus, the photogenerated holes and electrons are spatially separated, and subsequently the radiatve recombination becomes less favorable. On the other hand, with the hydrogen doped ZnO NWs, a flat band condition arises because of the small depletion width around surface due to an increase in the electron concentration. The enhanced spatial overlap of photogenerated carriers in the hydrogen doped $\mathrm{ZnO}$ NWs results in greater PL thermal quenching. The temperature-dependent PL intensity shown in Figure 2(b) provides additional evidence of hydrogen doping in the ZnO NWs.

\subsection{Time-resolved PL spectra of undoped and hydrogen doped ZnO NWs}

Figure 3(a) shows the PL decay profiles of the undoped and hydrogen doped ZnO NWs. The PL decay profiles are fitted using a biexponential function: $I(t)=A_{1} \exp \left(-t / \tau_{1}\right)+A_{2} \exp \left(-t / \tau_{2}\right)$ where $I$ is the PL intensity, $A$ is a pre-factor proportional to the intensity ratio, $\tau$ is the decay constant. For the undoped ZnO NWs, the shorter ( $\tau_{1}, 190 \mathrm{ps}$ ) and the longer ( $\tau_{2}, 420 \mathrm{ps}$ ) decay constants can be attributed to surface-related recombination and intrinsic radiative recombination processes, respectively. ${ }^{31}$ The PL decay profiles of hydrogen doped ZnO NWs with different plasma powers reveal shorter PL average lifetime ( $\left.\tau_{P L}\right)$, obtained from the weighted average of the decay constants: $\tau_{P L}=\left(A_{1} \tau_{1}^{2}+A_{2} \tau_{2}^{2}\right) /\left(A_{1} \tau_{1}+A_{2} \tau_{2}\right)$. To elucidate the origin of the PL decay dynamics decay constants, the decay 
profiles were analyzed as a function of the hydrogen plasma power. Figure 3(b) shows that the $\tau_{1}, \tau_{2}$, and $\tau_{P L}$ obtained at $3.362 \pm 0.003 \mathrm{eV}$ corresponding to the $\mathrm{I}_{4}$ emission energy at $10 \mathrm{~K}$ decreased as the plasma power increased. A higher plasma power may be expected to induce an increase in donor concentration due to the increase in the number of incorporated hydrogen atoms. The decrease in PL decay constants is consistent with other reports for doped semiconductors. ${ }^{32,33}$ The giant oscillator strength of bound excitons results in PL lifetime of bound excitons that is significantly shorter than that of free excitons. The increase in donor concentration leads to the formation of bound excitons via trapping of free excitons at donors. ${ }^{33}$ The flat band effect due to hydrogen doping (see Fig. 2(b)), affects the dominant radiative recombination path way. Figure 3(c) shows that the intensity ratio of the recombination pathway associated with $\tau_{1}$ increased as the temperature increased. This observation indicates that the surface-related recombination route becomes increasingly significant as the temperature increases. The absence of surface band bending at the surface of the hydrogen doped ZnO NW allows excitons to recombine through surface channels. Additionally, excitons can easily reach these surface channels since the typical exciton diffusion length in $\mathrm{ZnO} \mathrm{NW}$ is $150 \mathrm{~nm}$ at $10 \mathrm{~K}$, which is longer than the diameter of $\mathrm{ZnO} \mathrm{NW}(80 \mathrm{~nm})$ in this study. ${ }^{34}$

\section{Conclusions}

In summary, the effects of hydrogen plasma treatment on luminescent characteristics of ZnO NWs were investigated by temperature-dependent TIPL and TRPL measurements. The remarkable enhancement of the NBE emission of ZnO NWs through hydrogen plasma treatment indicates hydrogen doping in ZnO NWs. The recombination dynamics of donor bound excitons originating from hydrogen doping has been investigated by TRPL measurements along temperatures ranging from 10 to $100 \mathrm{~K}$. Significant decrease in the biexponential decay constants in the TRPL measurements was also observed. Additionally the absence of surface band bending in hydrogen doped $\mathrm{ZnO}$ nanowires makes surface-related recombination channels dominant. The combined study of TIPL and TRPL plays a crucial role to characterize luminescent properties of $n$ type $\mathrm{ZnO}$ NWs, which are essential for designing ultrafast X-ray imaging detectors.

\section{Acknowledgments}

This work was financially supported by Future-based Technology Development Program (Nano Fields) through the National Research Foundations (NRF) of Korea (0417-20140099). The work was performed in part at the Center for Integrated Nanotechnologies (CINT), a U.S. Department of Energy, Office of Basic Energy Sciences User Facility at Los Alamos National Laboratory (Contract DE-AC52-06NA25396) and Sandia National Laboratories (Contract DE-AC04-94AL85000). 
Prof. Joo acknowledges the support of the National Research Foundation of Korea (NRF) grant funded by the Korean government (MEST) (2011-0001215).
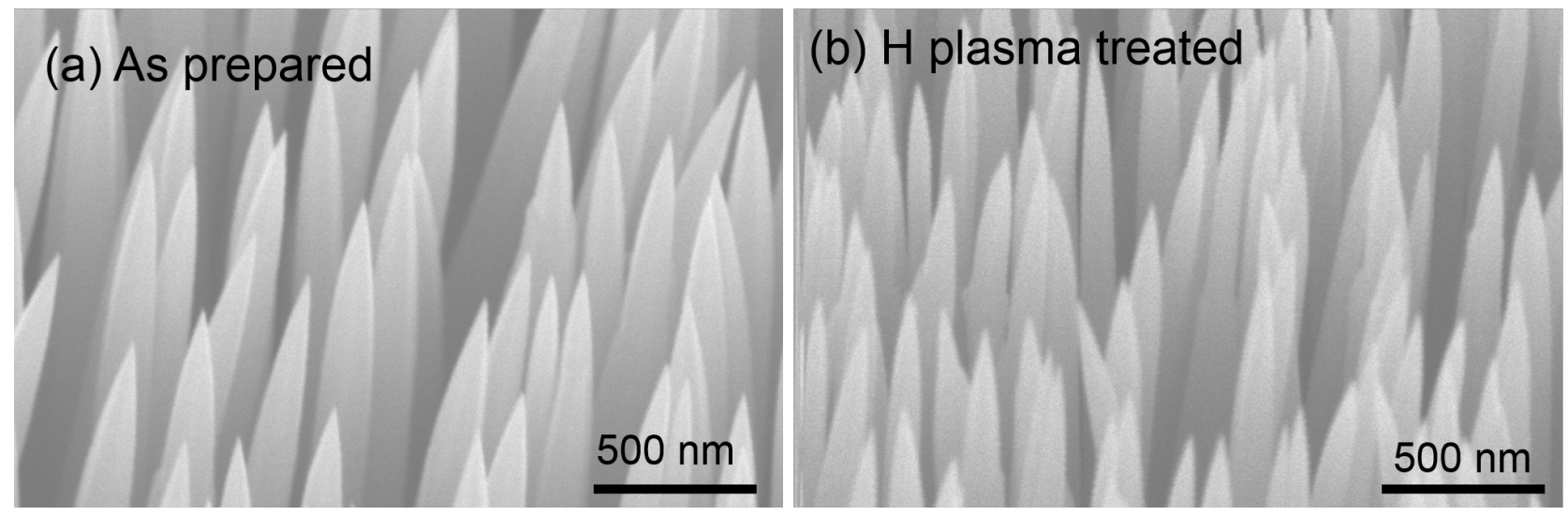

(c)

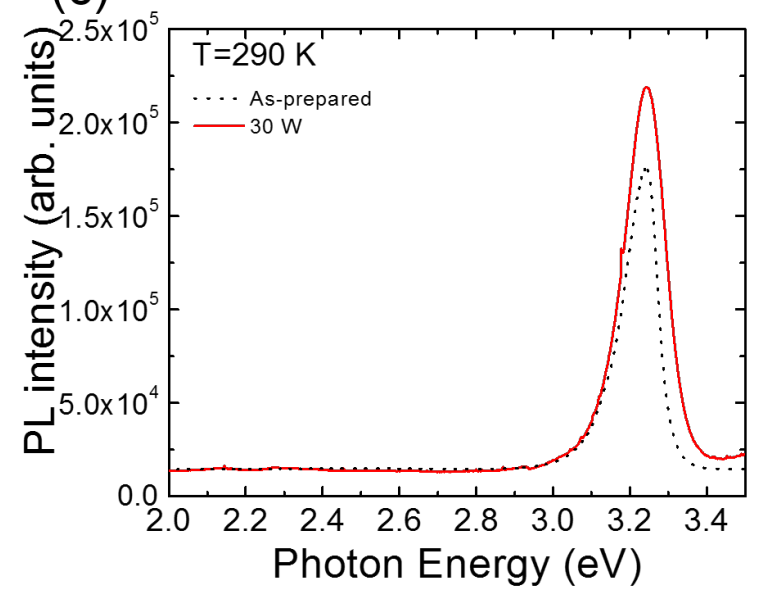

(d)

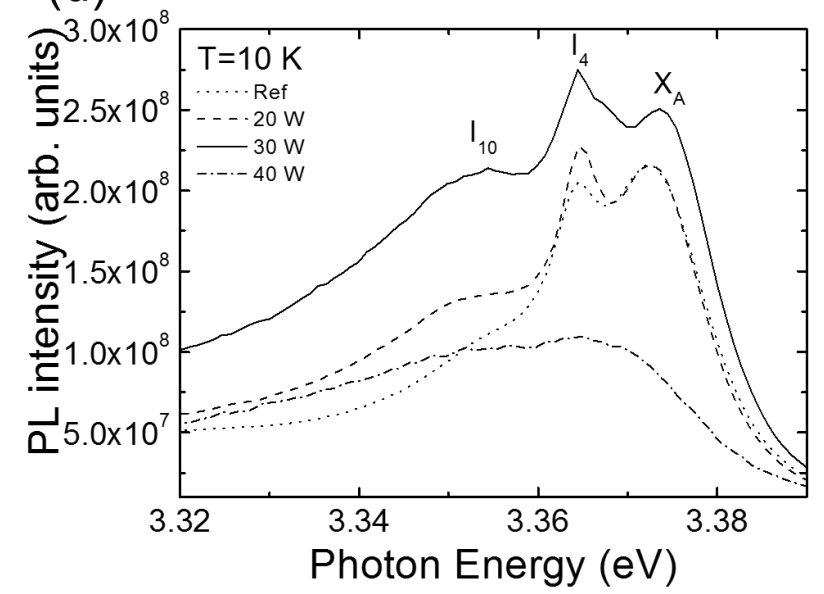

Figure 1. Scanning electron microscopy images of as-prepared (a) and of hydrogen plasma treated ZnO NWs with the plasma power of 30 W (b). (c) RT PL spectra of as-prepared (dotted, black) and hydrogen plasma treated (solid, red) ZnO NWs at $30 \mathrm{~W}$. (d) $10 \mathrm{~K}$ PL spectra of as-prepared (Ref) and hydrogen plasma treated ZnO NWs with different plasma powers. 

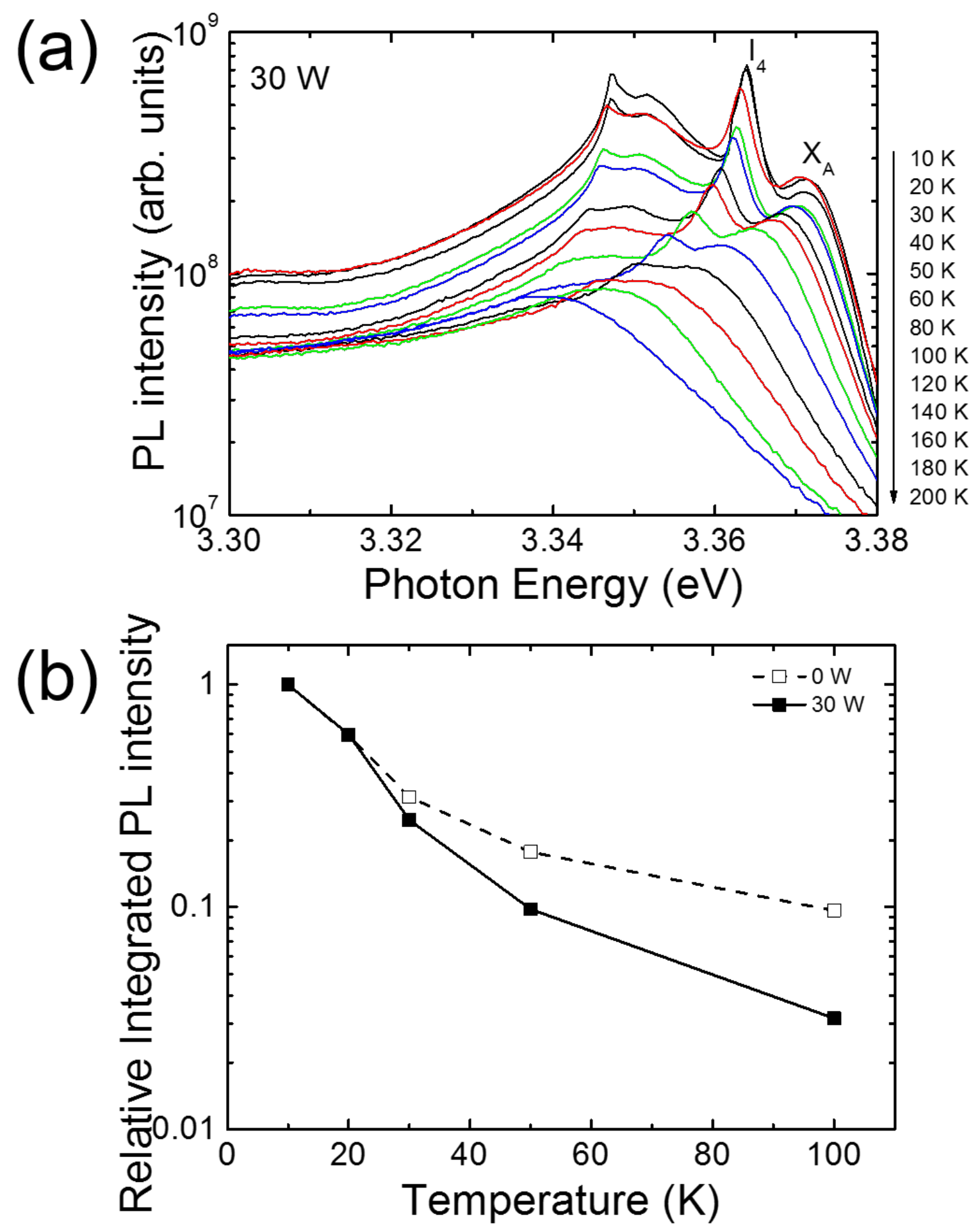

Figure 2. (a) Temperature-dependent PL spectra of hydrogen plasma treated ZnO NWs at $30 \mathrm{~W}$. (b) Relative integrated PL intensity of the NBE emission of as-prepared and hydrogen plasma treated ZnO NWs with the plasma power of $30 \mathrm{~W}$. The relative integrated PL intensity is the ratio of the integrated PL intensity at certain temperatures to that at $10 \mathrm{~K}$. 
(a)

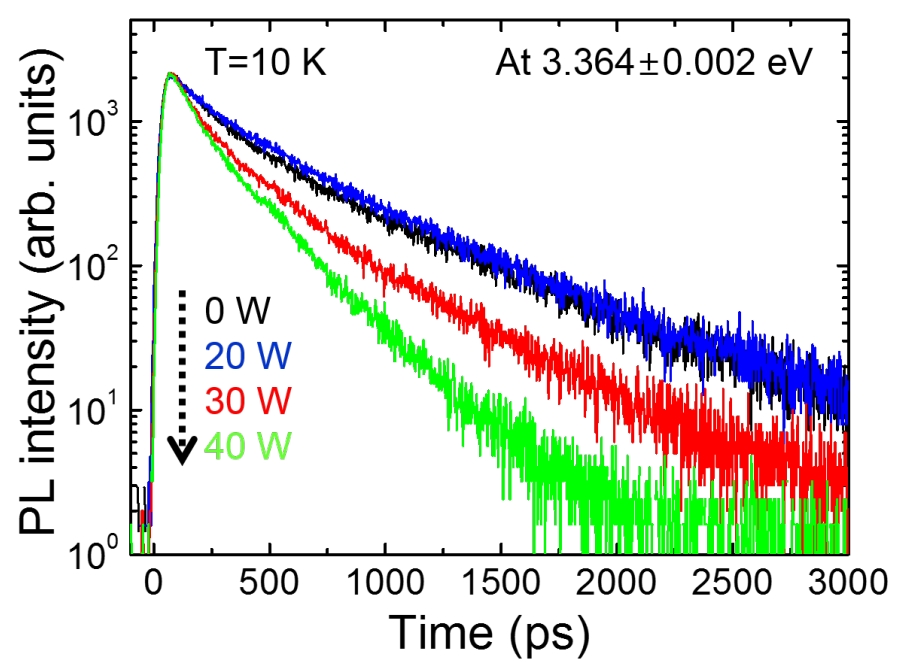

(b)

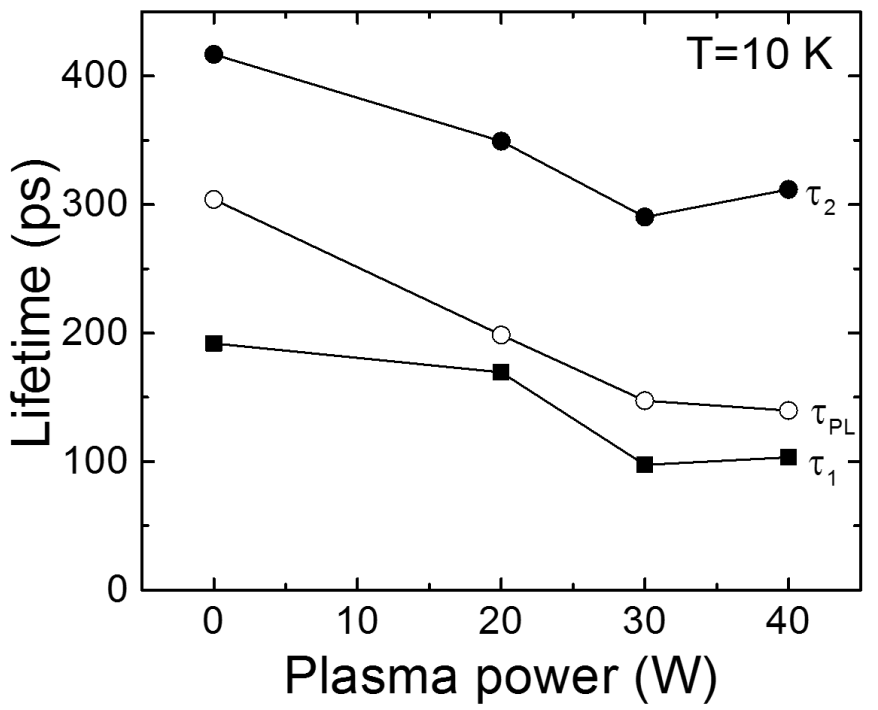

(c)

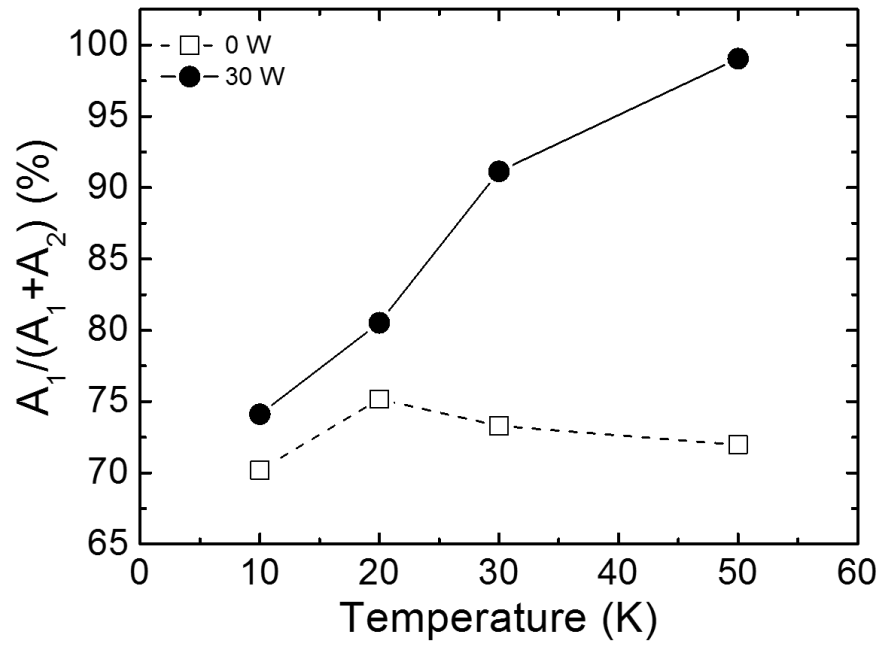

Figure 3. (a) The TRPL decay profiles at $10 \mathrm{~K}$ of as-prepared and hydrogen plasma-treated ZnO NWs with different plasma powers. (b) Plasma power-dependent PL decay constants and averaged PL lifetimes at $10 \mathrm{~K}$. (c) Temperature-dependent intensity ratios of the radiative recombination channels with the shorter decay constants of as-prepared and hydrogen plasma treated ZnO NWs at $30 \mathrm{~W}$. 


\section{References}

[1] C. L. Morris, N. S. P. King, K. Kwiatkowski, F. G. Mariam, F. E. Merrill, and A. Saunders, Rep. Prog. Phys. 76 (2013) 046301.

[2] A. Clarke, S. Imhoff, P. Gibbs, J. Cooley, C. L. Morris, F. E. Merrill, B. Hollander, F. G. Mariam, T. Ott, M. Barker, T. Tucker, W. K. Lee, K. Fezzaa, A. Deriy, B. Patterson, K. Clarke, J. Montalvo, R. Field, D. Thoma, J. Smith, and D. Teter, Sci. Rep. 3 (2013) 2020.

[3] http://science.energy.gov/ /media/bes/pdf/reports/files/NXD_rpt.pdf.

[4] W. Lehmann, Solid-State Electron. 9 (1966) 1107.

[5] D. Luckey, Nucl. Instrum. Meth. 62 (1968) 119.

[6] T. Batsch, B. Bengtson, and M. Moszyński, Nuclear Instruments and Methods 125 (1975) 443.

[7] Ali Taheri, Shahyar Saramad, and Saeed Setayeshi, Nucl. Instrum. Meth. A 701 (2013) 30.

[8] Ali Taheri, Shahyar Saramad, S. Ghalenoei, and Saeed Setayeshi, Rev. Sci. Instrum. 85 (2014) 013112.

[9] E. Bourret-Courchesne, S. E. Derenzo, and M. J. Weber, Nucl. Instrum. Meth. A 601 (2009) 358.

[10] S. E. Derenzo, M. J. Weber, E. Bourret-Courchesne, and M. K. Klintenberg, Nucl. Instrum. Meth. A 505 (2003) 111.

[11] Martin Nikl, Akira Yoshikawa, Anna Vedda, and Tsuguo Fukuda, J. Crys. Growth 292 (2006) 416.

[12] Guo-Dong Yuan, Wen-Jun Zhang, Jian-Sheng Jie, Xia Fan, Jian-Xin Tang, Ismathullakhan Shafiq, Zhi-Zhen Ye, Chun-Sing Lee, and Shuit-Tong Lee, Adv. Mater. 20 (2008) 168.

[13] Jinkyoung Yoo, Chul-Ho Lee, Yong-Joo Doh, Hye Seong Jung, and Gyu-Chul Yi, Appl. Phys. Lett. 94 (2009) 223117.

[14] Minjie Zhou, Haojun Zhu, Yang Jiao, Yangyan Rao, Suikong Hark, Yang Liu, Lianmao Peng, and Quan Li, J. Phys. Chem. C 113 (2009) 8945.

[15] Chris G. Van de Walle, Phys. Rev. Lett. 85 (2000) 1012.

[16] K. Ip, M. E. Overberg, Y. W. Heo, D. P. Norton, S. J. Pearton, C. E. Stutz, B. Luo, F. Ren, D. C. Look, and J. M. Zavada, Appl. Phys. Lett. 82 (2003) 385.

[17] Yuri M. Strzhemechny, John Nemergut, Phillip E. Smith, Junjik Bae, David C. Look, and Leonard J. Brillson, J. Appl. Phys. 94 (2003) 4256.

[18] Jinkyoung Yoo, Won Il Park, and Gyu-Chul Yi, J. Vac. Sci. Technol. B 23 (2005) 1970.

[19] Takashi Sekiguchi, Naoki Ohashi, and Yoshihiro Terada, Jpn. J. Appl. Phys. 36 (1997) L289.

[20] Naoki Ohashi, Takamasa Ishigaki, Nobuhiro Okada, Takashi Sekiguchi, Isao Sakaguchi, and Hajime Haneda, Appl. Phys. Lett. 80 (2002) 2869.

[21] Hooyoung Song, Jae-Hoon Kim, Eun Kyu Kim, and Sung-Min Hwang, Thin Solid Films 517 (2009) 3927.

[22] D. F. Wang, H. B. Lu, J. C. Li, Y. Wu, Y. Tian, and Y. P. Lee, Mater. Res. Bulletin 44 (2009) 41.

[23] A. Dev, R. Niepelt, J. -P. Richters, C. Ronning, and T. Voss, Nanotechnology 21 (2010) 065709.

[24] Cong Chen, Haiping He, Yangfan Lu, Kewei Wu, and Zhizhen Ye, Appl. Mater. Interfaces 5 (2013) 6354.

[25] W. I. Park, D. H. Kim, S.-W. Jung, and Gyu-Chul Yi, Appl. Phys. Lett. 80 (2002) 4232.

[26] E. V. Lavrov, J. Weber, F. Börrnert, Chris G. Van de Walle, and R. Helbig, Phys. Rev. B 66 (2002) 165205. 
[27] Yuri M. Strzhemechny, Howard L. Mosbacker, David C. Look, Donald C. Reynolds, Cole W. Litton, Nelson Y. Garces, Nancy C. Giles, Larry E. Halliburton, Shigeru Niki, and Leonard J. Brillson, Appl. Phys. Lett. 84 (2004) 2545.

[28] J. J. Dong, X. W. Zhang, J. B. You, P. F. Cai, Z. G. Yin, Q. An, X. B. Ma, P. Jin, Z. G. Wang, and Paul K. Chu, Appl. Mater. Interfaces 2 (2010) 1780.

[29] Cong Chen, Yangfan Lu, Haiping He, Mu Xiao, Zheng Wang, Lingxiang Chen, and Zhizhen Ye, Appl. Mater. Interfaces 5 (2013) 10274.

[30] B. K. Meyer, H. Alves, D. M. Hofmann, W. Kriegseis, D. Forster, F. Bertram, J. Christen, A. Hoffmann, M. Straßburg, M. Dworzak, U. Haboeck, and A. V. Rodina, Phys. Stat. Sol. (b) 241 (2004) 231.

[31] S. L. Chen, W. M. Chen, and I. A. Buyanova, Appl. Phys. Lett. 102 (2013) 121103.

[32] K. B. Nam, M. L. Nakarmi, J. Li, J. Y. Lin, and H. X. Jiang, Appl. Phys. Lett. 83 (2003) 2787.

[33] G. Pozina, S. Khromov, C. Hemmingsson, L. Hultman, and B. Monemar, Phys. Rev. B 84 (2011) 165213.

[34] Jinkyoung Yoo, Bonghwan Chon, Wei Tang, Taiha Joo, Le Si Dang, and Gyu-Chul Yi, Appl. Phys. Lett. 100 (2012) 223103. 